Livraisons

d'Histoire

de l'Architecture

\section{Livraisons de l'histoire de l'architecture}

$40 \mid 2020$

À propos des princes de Conti

\title{
150 ans plus tard, 150 mètres plus loin, la chapelle Saint-Ferdinand de Neuilly, un patrimoine déplacé
}

150 ans plus tard, 150 mètres plus loin, la chapelle Saint-Ferdinand de Neuilly, un patrimoine déplacé

150 years later, 150 meters further, the chapel of Saint-Ferdinand de Neuilly, a displaced heritage

Fünfzig Jahre später, fünfzig Meter weiter - die Umsetzung der Chapelle SaintFerdinand de Neuilly

\section{Justine Gain}

\section{OpenEdition}

\section{Journals}

Édition électronique

URL : http://journals.openedition.org//ha/1336

DOI : 10.4000/lha.1336

ISSN : 1960-5994

Éditeur

Association Livraisons d'histoire de l'architecture - LHA

Édition imprimée

Date de publication : 3 décembre 2020

Pagination : 99-111

ISSN : 1627-4970

Référence électronique

Justine Gain, «150 ans plus tard, 150 mètres plus loin, la chapelle Saint-Ferdinand de Neuilly, un patrimoine déplacé », Livraisons de l'histoire de l'architecture [En ligne], 40 | 2020, mis en ligne le 28 décembre 2020, consulté le 24 mars 2021. URL : http://journals.openedition.org//ha/1336 ; DOI : https://doi.org/10.4000//ha.1336 
Par Justine GAIN

\section{ANS PLUS TARD, 150 MÈTRES PLUS LOIN, LA CHAPELLE SAINT-FERDINAND DE NEUILLY, UN PATRIMOINE DÉPLACÉ}

Enclavée entre le périphérique parisien et la place du Général Konig, la chapelle Notre-Dame-de-la-Compassion contraste quelque peu dans un environnement caractéristique de la seconde moitié du XX $\mathrm{XX}^{\mathrm{e}}$ siècle. Vestige d'une monarchie oubliée, devenu paroisse ordinaire de quartier, quelle est l'histoire de cette chapelle à l'allure si singulière (ill. 1)?

\section{La mort du prince Ferdinand}

Hier, 13 juillet 1842, M. le duc d'Orléans est mort par accident ${ }^{1}$.

Le 13 juillet, vers 11 heures, Ferdinand-Philippe, héritier du trône échu à son père quelques années auparavant, quitte le palais des Tuileries, épicentre de la monarchie de Juillet. Il monte dans la voiture à la Daumont qui l'attend pavillon de Marsan et ordonne à son cocher de prendre la direction du château de Neuilly. Le prince désire faire ses adieux à la famille royale avant de se rendre au camp de Saint-Omer où il doit passer en revue plusieurs régiments. Destination finale : les eaux de Plombières où séjourne la duchesse Hélène, son épouse. À la hauteur de la porte Maillot, les chevaux du duc s'emballent à la vue d'un amoncellement de pierres destinées à l'érection de fortifications voisines. Le postillon est impuissant à retenir la voiture qui se précipite route de la Révolte. Le duc d'Orléans est violemment projeté au sol et le voici inconscient, agonisant sur le pavé. Il est rapidement transporté dans une habitation voisine pour recevoir les premiers soins. C'est là, dans l'arrière-boutique d'un certain Louis Lecordier, simple épicier et marchand de vin, que le prince décède quelques heures plus tard, à 4 heures de l'après-midi, au 4 route de la Révolte.

France, c'était ton bien c'était ton espérance :

Mais tout s'éclipse hélas! quand la mort a parlé ! ...

Il a contre une pierre heurté son existence,

Et son âme aussitôt vers les cieux a volé !!?

1. Victor Hugo, Choses vues, Paris, A. Michel, 1913, p. 70.

2. À la mémoire de Ferdinand-Philippe d'Orléans, estampe, Paris, vers 1842, BNF, Paris. 


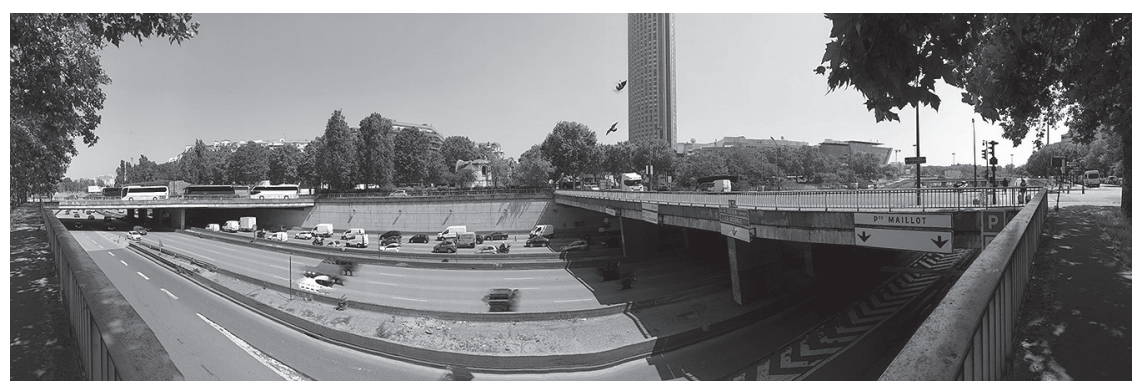

Ill. 1 : Vue de la chapelle Notre-Dame-de-la-Compassion depuis Neuilly-sur-Seine, 2019. (C) Grégoire Lubineau.

Après une brève annonce à la foule, Louis-Philippe ordonne le transfert du défunt au château de Neuilly. Le 30 juillet, un somptueux convoi emmène le corps à Notre-Dame-de-Paris pompeusement tendue de noir pour l'occasion. Officielles, les funérailles réunissent les personnages les plus importants du royaume. Le 4 août 1842, la dépouille du prince rejoint finalement le caveau familial à la chapelle de Dreux.

Au lendemain de la mort de l'héritier, d'autres dispositions sont prises route de la Révolte. Les pavés du chemin, maculés de sang, sont immédiatement remplacés sur ordres du roi ${ }^{3}$. Un mois plus tard, la maison de l'épicier est littéralement transportée dans le parc du château de Neuilly que feu M. le duc affectionnait particulièrement ${ }^{4}$. Aujourd'hui conservée au musée Carnavalet, une maquette reconstituant les lieux de l'accident est même réalisée, peut-être à l'initiative des souverains ${ }^{5}$. Enfin, Louis-Philippe et Marie-Amélie, terrassés par le chagrin, décident d'ériger une chapelle en mémoire de leur fils. Le souvenir du prince héritier de la Couronne s'incarnera en un lieu, en un monument matérialisant à jamais le drame royal.

\section{Sur les terres des Orléans}

Ainsi ces lieux, qui, par un cas fortuit, ont appartenu à S. M. Louis-Philippe, ont vu la mort de son fils aîné, et maintenant la reine des Français y fait élever une chapelle sous l'invocation du patron de son auguste fils : noble pensée d'une mère vertueuse ! ${ }^{6}$

À la fin du XVIII ${ }^{\mathrm{e}}$ siècle, Paris est prise en tenaille entre les murs des fermiers généraux. À l'ouest, la sortie de la ville s'effectue par la barrière de l'Étoile, l'actuelle porte Maillot, essentiellement constituée de terres en friche vaguement cultivées.

3. Victor Hugo, op. cit., p. 70.

4. La Phalange, dimanche 7 août 1842.

5. Henri Poncan, Maison où est mort le duc d'Orléans, le 13 juillet 1842, maquette, après 1842, PM37, Musée Carnavalet, Paris.

6. Description exacte de l'extérieur et de l'intérieur de la chapelle Saint-Ferdinand et de la chapelle de Dreux, Paris, Derche, [s.d.], p. 6. 
En 1815, Louis-Philippe acquiert le château de Neuilly qu'il ambitionne d'agrandir par l'achat de terrains alentour. Propriétaire d'une parcelle route de la Révolte - le futur numéro 4 -, il l'échange de bonne grâce contre un terrain agricole de taille équivalente près du château. La transaction effectuée, l'emplacement change une nouvelle fois de mains pour être cédé à un entrepreneur en maçonnerie, M. Parmentier. En 1812, le maçon demeurait sur la colline de Passy et y serait sans doute resté sans le projet avorté de Napoléon d'y faire construire le palais du Roi de Rome. En 1814, Parmentier s'installe 2 chemin de la Révolte dans une maison qu'il vient d'achever. La même année, à la chute de l'empereur, les soldats Alliées pillent et dévastent sur leur passage la modeste demeure. L'homme fait alors l'acquisition du terrain voisin, le 4 chemin de la Révolte, où son fils érige une maison occupée en 1842 par l'épicier Lecordier.

\section{Sauvegarder la mémoire royale}

Cette terre, autrefois propriété des Orléans, est de nouveau acquise pour le compte de la Couronne à la mort du prince Ferdinand-Philippe. Le comte de Montalivet, intendant de la Liste civile, est chargé de la transaction et le terrain est obtenu pour 110000 francs $^{7}$. C'est à Pierre Fontaine ${ }^{8}$, architecte du roi, que la conception des plans de la chapelle incombe. Après de dispendieuses premières esquisses, le projet se veut plus sobre. La première pierre est posée le 21 août 1842, un mois à peine après la mort du prince (ill. 2).

Les travaux, dans leurs aspects plus pratiques, sont confiés à Pierre-Bernard Lefranc ${ }^{9}$, ancien élève de Percier et Fontaine. Il est alors architecte du domaine privé du roi et contribue à l'édification de la chapelle en croix grecque, dans un style que l'on disait alors byzantin. Souhait ardent de la Reine, l'autel de la Vierge est disposé à l'endroit exact où son fils vient de mourir ${ }^{10}$. La sélection des artistes œuvrant sur le chantier est opérée avec la même attention, parmi les collaborateurs assidus de la famille royale. L'architecte Fontaine s'impose alors, de même que Lefranc, également concepteur de la chapelle des Orléans à Dreux. La décoration sculptée est confiée à Jean-Baptiste Plantar ${ }^{11}$, sculpteur des Bâtiments du roi, auteur de nombreux décors au château de Neuilly, au Louvre ou aux Tuileries. La maçonnerie est l'œuvre d'un certain Parmentier fils, qui sut savamment négocier l'obtention du chantier lors de la vente de sa parcelle. Ary Scheffer, professeur de dessin de la princesse Marie, esquisse les traits du monument funéraire dédié au

7. Description exacte [...], op. cit., p. 6.

8. Pierre Fontaine, Journal: 1799-1853, Paris, Imprimerie alençonnaise, 1987, Paris, t. 2, p. 1076.

9. Léon Charvet, Lyon artistique. Architectes : notices biographiques et bibliographiques avec une table des édifices et la liste chronologique des noms, Lyon, Bernoux et Cumin, 1899, p. 215.

10. Pierre Fontaine, op. cit., t. 2, p. 1076.

11. Justine Gain, Jean-Baptiste Plantar, le sculpteur ornemaniste de la Monarchie de Juillet, thèse de doctorat, sous la direction de Jean-Michel Leniaud et de Lionel Arsac, Paris, École pratique des hautes études et École du Louvre [en cours]. 


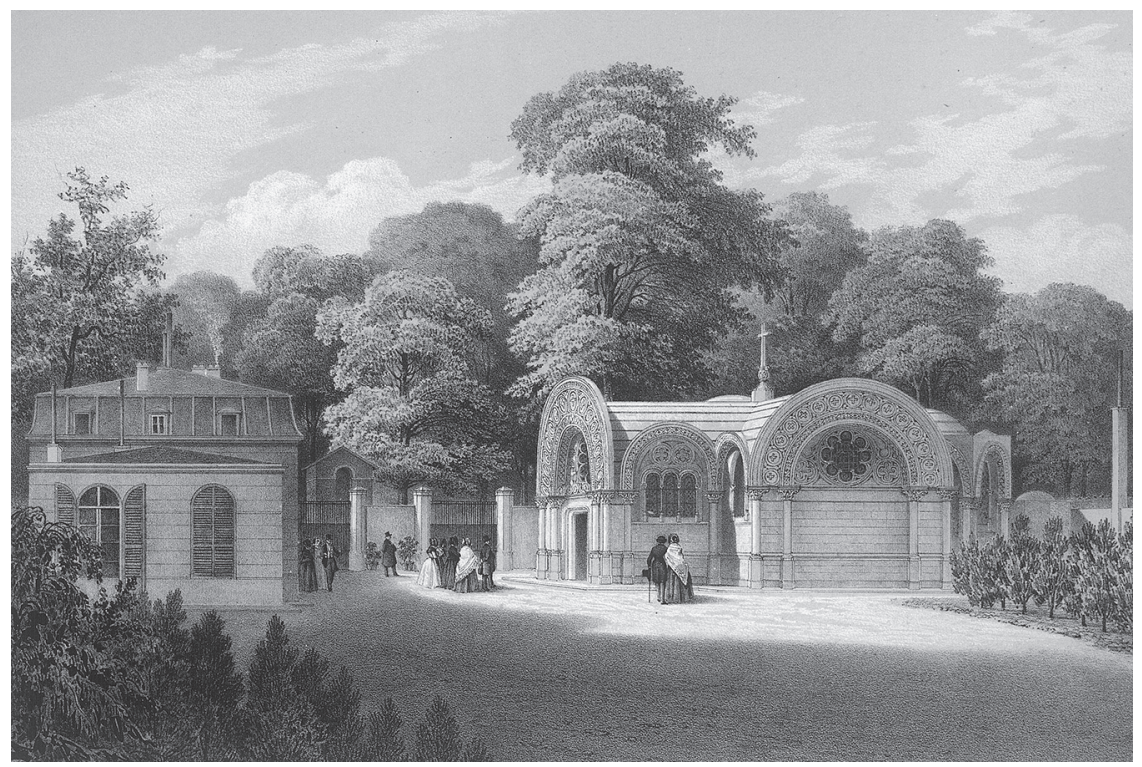

Ill. 2 : J.-B. Arnont, Vue de la chapelle Saint-Ferdinand à Neuilly, gravure, Paris, publié par F. Sinneltt, 10 Rotonde, Passage Colbert. (C) Collection privée.

prince dont Henri de Triqueti, familier du clan royal, sculpte le marbre ainsi que la Pieta, installée derrière l'autel principal. Le cénotaphe présente le prince allongé ; un ange, dessiné par Marie d'Orléans, s'agenouille au-dessus de son visage sans vie mais baigné de lumière. Celle-ci pénètre l'édifice à travers de somptueux vitraux manufacturés à Sèvres, d'après les dessins de Jean-Dominique Ingres, dont on connaît le célèbre portrait peint du prince Ferdinand.

À l'extérieur, la chapelle est flanquée de bâtiments annexes, lieux du service religieux, qui servent également d'appartements d'appoint à l'officiant et à la famille royale. Parés de noir, leurs intérieurs sont soigneusement aménagés et deux pendules

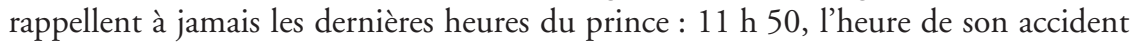
et $16 \mathrm{~h}$, celle de sa mort. Devant la chapelle, un espace est ménagé aux voitures arrivant de la route de la Révolte. Planté de quelques arbres préservant son intimité, le jardin est clos par deux grilles, jalousement gardées par l'ancien épicier Lecordier, désormais gardien du cénotaphe royal.

Un an après les faits, le 11 juillet 1843 , à quelques jours de l'anniversaire de la mort du duc, la chapelle est inaugurée. La Reine s'y recueillera dorénavant chaque mercredi, preuve supplémentaire de la préoccupation toute personnelle qui préside à l'aménagement de ce lieu, à la dimension tant géographique qu'émotionnelle. 


\section{Un territoire fortifié}

Désormais empreinte d'une importante charge mémorielle, la route de la Révolte est aussi partie prenante d'un ensemble urbain stratégique, aux portes de Paris. L'amas de pierres, à l'origine supposée de l'accident du duc d'Orléans, était en réalité destiné à l'érection de la nouvelle « enceinte Thiers " appelée à se dresser autour de la capitale.

Au fil des siècles, se sont succédés à Paris plusieurs dispositifs défensifs, depuis les enceintes gallo-romaines du $\mathrm{IV}^{\mathrm{e}}$ siècle jusqu'au mur des fermiers généraux ${ }^{12}$. Par crainte de nouvelles exactions alliées, le projet d'une nouvelle enceinte émerge progressivement. En 1839, une pétition est présentée en ce sens au gouvernement, ardemment défendue par le duc d'Orléans ${ }^{13}$ et son aide de camp François de ChabaudLatour ${ }^{14}$. L'idée est alors portée par Adolphe Thiers, éphémère président du Conseil, et la loi promulguée le 3 avril 1841. Les travaux s'échelonnent entre septembre 1841 et $1845^{15}$. Chabaud-Latour est d'ailleurs chargé de veiller à l'édification est de l'enceinte qui doit prévenir de l'arrivée d'ennemis extérieurs mais se targue aussi de pouvoir museler d'éventuels soulèvements populaires, si récurrents depuis 1789 .

Les fortifs comme on les appelle alors, enserrent Paris. Une large route, la rue militaire, est doublée d'un épais mur ponctué de bastions et de portes. Construite au-delà des limites administratives de 1841, l'enceinte s'étend sur les communes limitrophes. Les faubourgs de la ville - alors situés en dehors de celle-ci - sont donc protégés avant d'être officiellement annexés à la capitale en 1860. Un glacis délimite une zone de servitude, dite de non aedificandi, sur laquelle aucune construction durable ne doit, en théorie, être édifiée. En cas de conflit, la zone peut être arasée dans un rayon de $250 \mathrm{~m}$. Seules les structures légères pouvant être facilement démolies sont permises, exception faite de la chapelle dont la parcelle est directement bornée par l'enceinte. Lorsque Paris s'agrandit en 1860, les rues militaires deviennent les boulevards des maréchaux enveloppant la ville redessinée. Ainsi, se cristallisent autour des fortifications Thiers d'importants enjeux stratégiques et militaires dont la chapelle devra largement pâtir.

Le 29 juillet 1870, la France déclare la guerre à la Prusse. Le siège de la capitale menace et le 12 août ${ }^{16}$, les mesures d'urgence concernant l'enceinte sont mises en œuvre par le général Trochu, gouverneur de Paris. Les habitants de la zone militaire sont sommés de quitter les lieux.

12. Marie-France Sardain, "Les servitudes militaires autour des fortifications au XIX ${ }^{\mathrm{e}}$ siècle ", Revue historique des armées, 2014, 274, [En ligne] http://journals.openedition.org/rha/7940 (consulté le 11 août 2020).

13. Les fortifications en Île-de-France, 1792-1844, étude de l'Institut d'Aménagement et d'Urbanisme de la Région d'Île-de-France et de l'Association Vauban, juin 2005, p. 36.

14. Chabaud-Latour (1804-1885) a été l'aide de camp du duc d'Orléans jusqu'à sa mort en 1842.

15. Jean-Louis Cohen, Des fortifs au périph. Paris, les seuils de la ville, Paris, Picard éditeur, 1991, p. 31-33.

16. Il s'agit de la loi du 12 août 1870 par laquelle le général Trochu, gouverneur de Paris et président de la Défense nationale, ordonne l'évacuation de la zone de non aedificandi autour des remparts. 


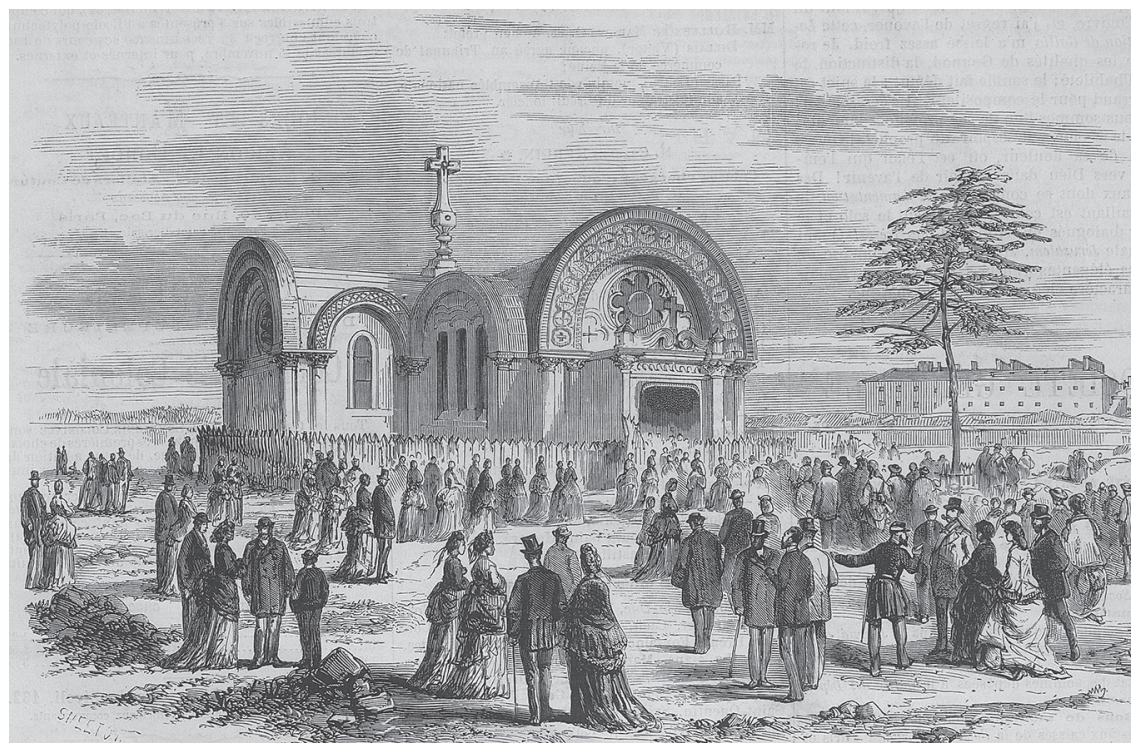

Ill. 3 : L'Illustration, 18 novembre 1871, p. 336.

La zone militaire des fortifications, entièrement démolie et rasée offre des perspectives toutes nouvelles. On aperçoit sur la droite, en sortant, la chapelle commémorative construite à la place où est mort le duc d'Orléans, sur le chemin de la Révolte. Sans doute elle a obtenu grâce comme monument historique; d'ailleurs sa forme basse se rapprochant de celle d'un tombeau n'exigeait pas impérieusement qu'on la sacrifiât ${ }^{17}$.

Tout ce qui encombre la zone militaire et compromet la bonne défense de la capitale est abattu, exception faite une nouvelle fois de la chapelle heureusement épargnée par l'intervention du général de Chabaud-Latour ${ }^{18}$. Compagnon d'armes du duc d'Orléans, il participe au projet des fortifs comme député du Gard. Rappelé aux responsabilités militaires pour contribuer à la défense de Paris en 1870, il prend la présidence du comité des fortifications, position dont il use pour protéger la chapelle honorant la mort de son vieil ami.

Si la bâtisse principale est sauvée, les appartements ${ }^{19}$ attenants, l'entrée ainsi que le jardin, sont détruits courant septembre $1870^{20}$ sur décision du génie militaire français. La chapelle est sommairement entourée de palissades de fortune ; les arbres et autres habitations environnants sont systématiquement abattus (ill. 3).

17. Théophile Gautier, Tableaux de Siège, 1870-1871, Paris, Charpentier et C ${ }^{\text {ie }, 1871, ~ p . ~} 97$.

18. Pierre Wachenheim, Le $17^{e}$ arrondissement, itinéraires d'histoire et d'architecture, Paris, Action artistique de la Ville de Paris, 2000, p. 62. Médiathèque de l'Architecture et du Patrimoine (MAP), Charenton, 1995/025/0893.

19. L'Illustration, 18 novembre 1871 , p. 336.

20. Monde illustré, 24 septembre 1870. 
Durant le siège, notamment en janvier 1871, l'entrée ouest de Paris est sévèrement bombardée par les forces prussiennes. Lorsque la guerre s'achève la chapelle est toujours debout, à son emplacement d'origine, mais sa disposition initiale a amplement souffert des combats et la Porte Maillot n'est plus qu'un champ de ruines à l'ouest de Paris.

\section{De 1870 à 1950, entre pauvreté et divertissements}

Dans les années 1870, les remparts de la Porte Maillot sont partiellement détruits et deviennent peu à peu le lieu de constructions éphémères. Certains témoignent d'une pauvreté parisienne rejetée aux portes de la ville et auparavant concentrée dans les faubourgs extérieurs du mur des fermiers généraux ${ }^{21}$. La Zone, cet « espèce de village qui n'arrive jamais à se dégager tout à fait de la boue, coincé dans les ordures et bordé de sentiers où les petites filles trop éveillées et morveuses, le long des palissades, fuient l'école pour attraper, d'un satyre à l'autre, vingt sous, des frites et la blennorragie " ${ }^{22}$, est occupée par une population indigente pour qui Paris intramuros reste inaccessible et les bâtisses insalubres l'unique logement envisageable ${ }^{23}$.

Dans un contraste saisissant de proximité, les entrées de la ville se parent bientôt de tous les atours du divertissement. Hors de Paris, exemptés de l'octroi, bals, cinémas, foires, expositions et autres buvettes saisonnières s'établissent temporairement sur les zones de non aedificandi. La Porte Maillot n'échappe pas à la règle et autour de la chapelle du duc d'Orléans se succèdent plusieurs établissements.

À l'angle de la route de la Révolte et de l'avenue de la Grande Armée, l'aérodrome de la Porte Maillot voit le jour dans les années 1890. Un théâtre géant, le Columbia ${ }^{24}$, élit également domicile sur l'un des terrains vagues jouxtant le cénotaphe. Le directeur, Bolossy Kiralfi est l'un des grands personnages du monde du spectacle de la Belle Époque. L'Exposition Universelle de $1900^{25}$ est l'occasion de promouvoir cet espace installé sur plus de $10000 \mathrm{~m}^{2}$ et abritant un cirque aquatique, un hippodrome et pouvant accueillir jusqu'à 6000 spectateurs. Finalement détruit, le théâtre laisse place au Printania ${ }^{26}$, lieu pour "tous les vrais Parisiens en quête de plaisir ${ }^{27}$, qui ouvre ses portes le 10 juin $1904^{28}$. Ce "jardin concert-music-hall» offre tout un panel de spectacles, bars, toboggans et autres plaisirs en vogue. Paul Ruez, son directeur, est également propriétaire du Parisiana, haut lieu du divertissement des années 1900 .

21. Les fortifications en Île-de-France, 1792-1844, étude de l'Institut d'Aménagement et d'Urbanisme de la région d'île-de-France et de l'Association Vauban, juin 2005, p. 78.

22. Louis-Ferdinand Céline, Voyage au bout de la nuit, Paris, 1932.

23. Dans son court métrage éponyme, George Lacombe illustre ce dénuement des faubourgs en 1928.

24. Théatre géant "Columbia" à la Porte Maillot, affiche, Paris, Imprimerie Chardin, 1890, Paris.

25. "À la Porte Maillot ", Le Monde illustré, 22 juillet 1899, p. 78.

26. Pierre Wachenheim, op. cit., p. 45.

27. "Réouverture du Printania ", Comoedia, 31 mai 1908, p. 3.

28. "Printania ", Revue illustrée, 15 juin 1904. 
Tout ce que Paris compte de notabilités mondaines, artistiques, littéraires, etc, tout ce que Paris compte d'étrangers (demandez aux agents polyglottes) était à la réouverture de Printania ${ }^{29}$.

Sur ce même emplacement, le Luna Park, inauguré le 29 mai 1909, succède au Printania. Inspiré de son homologue new-yorkais de Coney Island, le parc offre de nouveaux divertissements. Quant à la chapelle, elle est désormais ceinturée de montagnes russes et autres attractions, pour le plus grand amusement des parisiens en goguette.

Puis, tout à coup, naquit l'Uni-Park [Luna Park], avec la violence de ses bruits, l'insolence de ses clameurs. C'était une honte, me disais-je, d'installer un lieu de plaisir aussi près d'une tombe ${ }^{30}$.

Le parc est finalement détruit en 1948 et le site dévoué aux nouveaux aménagements urbains qu'exige une quête soudaine et effrénée de modernité, au commencement des Trente Glorieuses.

\section{La Porte Maillot, cour de la modernité}

L'aménagement de la Porte Maillot et le sort de la chapelle qui lui est associé ne s'appréhende qu'à travers une considération élargie d'un Paris en perpétuelle évolution au cours du $\mathrm{XX}^{\mathrm{e}}$ siècle. Déjà jugée obsolète par certains lors de sa construction, l'enceinte est l'objet d'un vif débat autour du bien-fondé de sa conservation à l'heure d'une mutation drastique de l'équipement et des techniques militaires et alors même que la défense de Paris a été entièrement repensée en 1874 par le général Séré de Rivière ${ }^{31}$. Les nouvelles formes de combat de la guerre 1914-1918 abondent dans le sens d'un déclassement de l'enceinte Thiers, confirmé par la loi du 19 avril 1919. Progressivement détruits jusqu'en 1929, les remparts rendent disponible une immense friche que l'on propose de couvrir d'infrastructures, d'espaces verts et de logements bon marché, solutions adaptées aux besoins d'une ville alors en pleine croissance.

Dans cette optique, est lancé en 1931 le concours Rosenthal. Inspirés par un américanisme latent, les architectes les plus réputés - Le Corbusier, Henri Sauvage ou Auguste Perret - proposent une porte Maillot radicalement moderne ${ }^{32}$. En avril 1933, un avant-projet d'aménagement est même discuté au Conseil municipal de Paris sans qu'aucune suite ne lui soit donnée. En 1928, le bail du Luna Park est même reconduit pour 30 ans.

29. Ibid.

30. Raymond Queneau, Pierrot mon ami, Paris, Gallimard, 1842, p. 79. Le roman se déroule dans un décor largement inspiré de celui de la Porte Maillot dans les années 1930.

31. Les fortifications de Paris en Ile-de-France, op. cit., p. 99.

32. Jean-Louis Cohen, op. cit., p. 218. 
Pourtant, la porte Maillot est bien un carrefour crucial de la capitale notamment en ce qui concerne la circulation automobile : elle constitue le point d'entrée majeur dans la ville par l'ouest. Alors que les constructions haussmanniennes de l'avenue de la Grande Armée se heurtent aux fantômes des remparts et à l'orée verte du bois de Boulogne, la nécessité de nouvelles installations fonctionnelles se fait sentir. Dans les années 1930, les propriétaires de la chapelle Saint-Ferdinand de Neuilly apprécient parfaitement la situation et prennent, en conséquence, les dispositions nécessaires. Déjà inscrite sur l'inventaire supplémentaire des Monuments historiques depuis le 5 juin $1928^{33}$, la chapelle réclame, selon eux, davantage de protection.

La Société civile du Domaine de Dreux souhaiterait que cette protection fût plus effective et plus durable; la Chapelle est, en effet, située sur la zone "non aedificandi » et, pour ce motif, exposée à être démolie si la Ville de Paris, en raison d'un objectif que l'on peut prévoir, prenait cette décision ${ }^{34}$.

Elle est définitivement classée le 15 décembre $1928^{35}$ afin que la "protection de l'État s'exerce " ${ }^{36}$ de manière optimale. Cette demande avisée sauvera, pensent-ils, la chapelle d'une destruction programmée mais ne préservera malheureusement pas son agencement initial, déjà largement mutilé en 1870 .

\section{"Paris, Porte Maillot, ce qui fut autrefois Lunapark n'est plus " ${ }^{37}$}

Après les affres de la Seconde guerre mondiale, Paris s'imagine en cité ultra moderne. En 1954, les préconisations de Bernard Lafay ${ }^{38}$ sont adoptées au Conseil municipal de Paris ${ }^{39}$. L'objectif est simple: une politique de circulation marquée grâce à des aménagements urbains conséquents tels que la construction du périphérique. À la fin des années 1960, la question de l'aménagement de la Porte Maillot est à nouveau posée. Le projet d'un "Hôtel-Palais des Congrès » voit le jour et s'accompagne d'une nouvelle conception de la mobilité automobile. Initié par la

33. MAP, D/1/75/231-2, arrêté inscrivant la chapelle Notre-Dame-de-la-Compassion sur l'inventaire supplémentaire des Monuments historiques, 5 juin 1928.

34. MAP, Charenton, D/1/75/231-2, lettre du directeur de la Société civile du domaine de Dreux au directeur général des Beaux-Arts, 12 juillet 1928.

35. MAP, Charenton, D/1/75/231-2, arrêté de classement de la chapelle Notre-Dame-de-la-Compassion, 15 décembre 1928.

36. MAP, Charenton, D/1/75/231-2, rapport de Paul Boeswillwald sur la demande de classement de la chapelle Notre-Dame-de-la-Compassion, 15 décembre 1928.

37. "La Porte Maillot tournera le dos à Lunapark », Le Journal de Paris, 4 mai 1971.

38. Jean-Louis Cohen, op. cit., p. 256. Bernard Lafay, "La circulation ", Solutions aux problèmes de Paris, Paris, Imprimerie de Desfossés, 1954.

39. Nathalie Roseau, "L’infrastructure sismographe. Temps, échelles et récits du boulevard périphérique parisien ", Tracés. Revue de Sciences humaines, 35, 2018, [En ligne] http://journals.openedition.org/ traces/8207 (consulté le 10 août 2020). 
Chambre d'Industrie et de Commerce de Paris, ce nouveau projet s'étendra sur le terrain jadis occupé par le Luna Park et depuis employé comme parc automobile par le Ministère de l'Intérieur ${ }^{40}$. Il se conçoit d'abord sans se soucier de l'existence presque oubliée de la chapelle de la Compassion. L'érection d'une tour devant abriter près d'un millier de chambres d'hôtel fait d'ailleurs l'objet d'un débat esthétique. Celle-ci ne devra pas obstruer la perspective de l'axe Arc de Triomphe-Louvre - «On ne peut que craindre la perte d'un effet mondialement connu ${ }^{41}$ " - et donc prendre place sur les lieux de l'accident du duc d'Orléans ${ }^{42}$. Les déplacements routiers et piétons préoccupent également ; les autorités et les concepteurs du projet s'y prennent à plusieurs reprises avant de statuer sur le tracé final. Le sort de la chapelle ne semble pas inquiéter outre mesure malgré les nombreuses commissions - urbanisme, monuments historiques, permis de construire - qui statuent sur le projet entre 1965 et 1972. Lors de la Conférence permanente du permis de construire du 22 novembre 1968, le "problème " de la chapelle, sise sur le terrain destiné au projet, est enfin soulevé :

Le Ministère des Affaires culturelles a émis un avis défavorable en raison de la suppression de la chapelle de la Compassion Boulevard Pershing. Mais celle-ci serait reportée à l'angle de la Porte des Ternes et du boulevard Aurelle de Paladines. Dans ces conditions, il semble que satisfaction soit donnée au service des Monuments Historiques ${ }^{43}$.

Impossible de rayer de la carte la chapelle classée Monument historique, l'édifice sera donc délogé ${ }^{44}$. L'aval de la Société civile du Domaine de Dreux, propriétaire effectif, est indispensable mais s'obtient aisément en échange de l'installation d'une plaque commémorative à l'endroit même de l'accident, au sein du futur hôtel ${ }^{45}$.

L'immeuble classé ne peut être détruit ou déplacé, même en partie, ni être l'objet d'un travail de restauration, de réparation ou de modification quelconque, si le ministre chargé des affaires culturelles n'y a donné son consentement ${ }^{46}$.

40. MAP, Charenton, 2001/23/26, lettre d'André Jean Donzet à l'ingénieur général du service des parcs et jardins, $1^{\text {er }}$ décembre 1966.

41. MAP, Charenton, 1991/025/004, lettre de l'adjoint à l'Inspection générale des Monuments historiques au ministre des Affaires culturelles, 25 novembre 1968.

42. Arch. Nat., Pierrefitte, 19960493/69.

43. MAP, Charenton, 1991/025/004, lettre de l'adjoint à l'Inspection générale des Monuments historiques au ministre des Affaires culturelles, 25 novembre 1968.

44. MAP, Charenton, 2001/023/0026, lettre de Bazelaire de Rupierre, architecte du comte de Paris, à Bernard Vitry, architecte en chef des Monuments historiques, 13 octobre 1966.

45. MAP, Charenton, 2001/023/0026, rapport de Bazelaire de Rupierre sur la chapelle Notre-Damede-la-Compassion, 15 juin 1968.

46. Article 9 de la loi du 31 décembre 1913 dans sa version en vigueur du 4 janvier 1914 au 13 juillet 1985. 


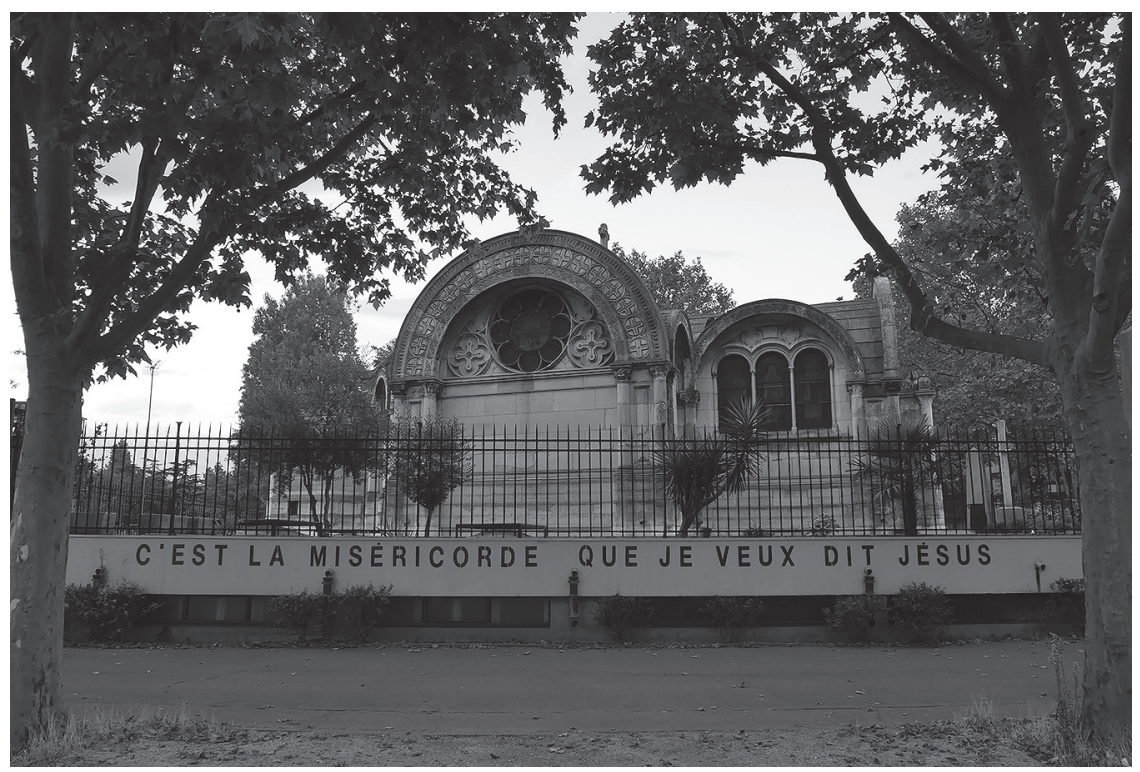

Ill. 4 : Vue de la chapelle Notre-Dame-de-la-Compassion à Paris, 2020. (C) Justine Gain.

Après l'accord des différentes parties ${ }^{47}$ et malgré le " mauvais état " de conservation de l'édifice ${ }^{48}$, le déplacement de la chapelle est finalement acté le 11 décembre 1968 par André Malraux, alors ministre des Affaires culturelles ${ }^{49}$. Entre 1970 et début 1971, la chapelle est de ce fait déplacée, "pierre par pierre " ${ }^{50}$, à 150 mètres du lieu de sa création, sur un terrain gracieusement offert par la ville de Paris à l'angle du boulevard Aurelle-de-Paladine et de la Porte-des-Ternes, en surplomb du futur périphérique ${ }^{51}$.

À tous les égards, l'intérêt de la chapelle Notre-Dame-de-la-Compassion n'est pas d'une importance suffisante pour justifier son maintien sur place et son incorporation dans la nouvelle composition, serait-ce en invoquant l'illustre exemple de Manhattan ${ }^{52}$.

47. MAP, Charenton, 2001/023/0026, compte rendu de la réunion à propos du transfert de la chapelle Saint-Ferdinand, 23 avril 1968.

48. MAP, Charenton, 1991/025/0004, lettre du ministre d'État chargé des Affaires culturelles au préfet de Paris, 27 septembre 1967.

49. MAP, Charenton, 1991/025/004, lettre du ministre d'État chargé des Affaires culturelles au préfet de Paris, 15 janvier 1969.

50. MAP, Charenton, 2001/023/0026, notice descriptive des travaux à exécuter à la chapelle NotreDame-de-la-Compassion, 17 juin 1968.

51. "Paris - district - Boulevard Pershing 200 mètres pour une chapelle ", La Croix, 23 septembre 1970.

52. Arch. nat, 19960493/69, lettre de Jean-Pierre Paquet, adjoint à l'Inspection générale des Monuments historiques, au ministre des Affaires culturelles, 15 novembre 1968. 


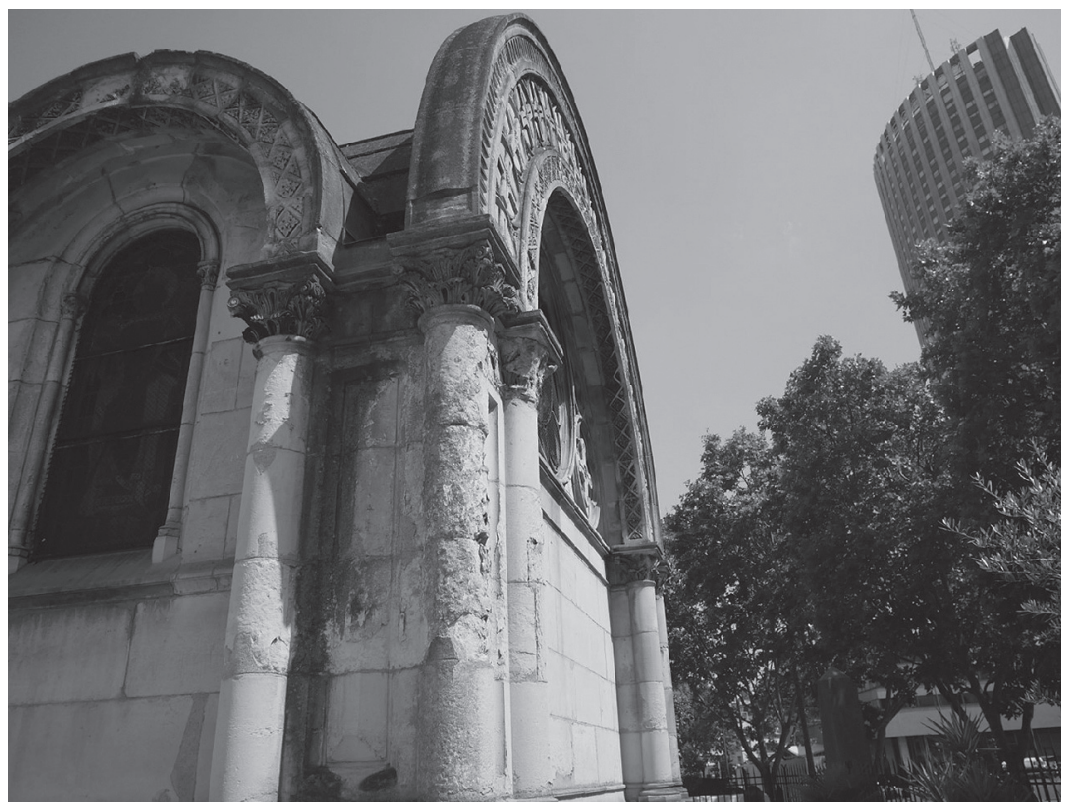

Ill. 5 : Vue du côté ouest de la chapelle Notre-Dame-de-la-Compassion à Paris, 2020. (C) Justine Gain.

Olivier-Pomponne Bazelaire de Rupierre, architecte du comte de Paris, est chargé du transfert ${ }^{53}$. À cette occasion, un sous-sol flambant neuf est aménagé afin d'accueillir célébrations et activités paroissiales en tout genre ${ }^{54}$. Le coût de l'opération, avoisinant le million de francs, est soutenu par la Chambre de Commerce ${ }^{55}$ et, dans une moindre mesure, par l'archevêché de Paris s'agissant des salles souterraines. La presse se fait d'ailleurs l'écho de la prouesse technique que représente alors le déplacement de la chapelle : "Comme quoi le Paris d'hier et celui de demain peuvent cohabiter pour peu qu'on le veuille " ${ }^{56}$ ! En 1972, André-Jean Donzet, architecte en chef des Monuments historiques, alerte ${ }^{57}$ sur l'état critique de la chapelle ainsi que sur l'urgence d'une restauration, notamment extérieure.

Cependant, il y a lieu de noter que l'importance des travaux de sculpture est sans commune mesure avec la vétusté du monument, et que les travaux auraient été bien moindres (à supposer qu'ils aient été nécessaires) si les opérations de démontage et remontage n'avaient pas eu lieu ${ }^{58}$.

53. "Une chapelle parisienne déplacée pierre par pierre ", Le Figaro, 9 octobre 1970.

54. "Reconstruit pierre par pierre", L'Aurore, 6 mai 1971.

55. MAP, Charenton, 2001/023/0026, convention pour le transfert de la chapelle Notre-Dame-dela-Compassion entre la chambre de Commerce et de l'Industrie de Paris et la Société civile du Domaine de Dreux.

56. "Paris - district - Boulevard Pershing 200 mètres pour une chapelle ", op. cit.

57. MAP, Charenton, 1991/025/0004, rapport présenté par l'architecte en chef des Monuments historiques à l'appui d'un projet de travaux ayant pour objet la remise en état du décor extérieur.

58. Ibid. 


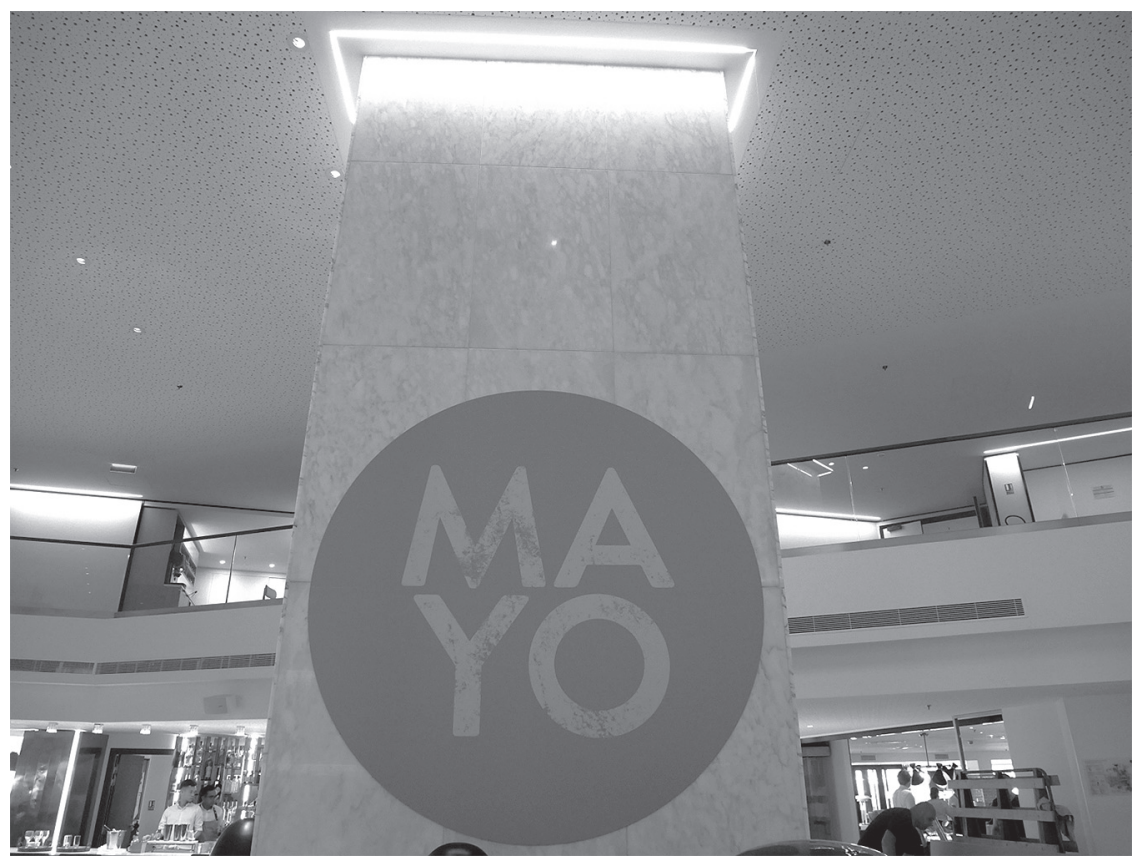

Ill. 6: Vue du Hall de l'hôtel Hyatt, 2019. (C) Justine Gain.

Malgré sa restauration durant l'année $1974^{59}$, la chapelle reste aujourd'hui dans un état de conservation préoccupant dans un environnement urbain peu propice à l'amélioration de son état (ill. 4 et 5). Témoignage unique d'un lieu pensé par une famille endeuillée, la plaque commémorative exigée par les héritiers en échange du déplacement de la chapelle est désormais dissimulée sous l'immense autocollant vert pomme - parés des quelques lettres Mayo - invitant les clients de l'hôtel Hyatt à se rassasier à la table d'un restaurant contemporain (ill. 6).

Porte Maillot, de nouvelles transformations sont prévues par la ville de Paris autour de la chapelle de la Compassion ${ }^{60}$. Une extension du bois de Boulogne ainsi que de nouveaux immeubles végétalisés entoureront bientôt le cénotaphe du $\mathrm{XIX}^{\mathrm{e}}$ siècle, l'enserrant dans un étau urbain toujours plus anachronique et questionnant encore la préservation des monuments historiques et de leurs abords.

Justine GAIN

Doctorante en histoire de l'art (EPHE), chargée d'études et de recherche à 
